\title{
Atrial fibrillation: an epidemiologic, scientific and clinical challenge
}

\author{
Valentin Fuster
}

Atrial fibrillation (AF) has been classified into three main types: paroxysmal AF is intermittent, recurrent and usually self-terminating, with durations of 2 min to 7 days; persistent AF usually lasts more than 7 days and can be therapeutically cardioverted to sinus rhythm; permanent AF cannot be terminated by cardioversion or has been present for more than 1 year without attempted cardioversion. Advances in our understanding of AF can be described metaphorically by these classifications. From 1991, when the Framingham Heart Study disclosed the significant rate of $A F$ in their population, to 2001 , data were paroxysmal, with various important characteristics of AF beginning to recur in the literature. Since 2001, and after the publication of the ACC/AHA/ESC management guidelines, AF has become a persistent and permanent challenge, and four critical features deserve comment.

First, prevalence and cost are rising. AF prevalence in North America and Western Europe ranges from almost $1 \%$ at $\leq 60$ years to around $10 \%$ in people $>80$ years. Hospital admission rates for $\mathrm{AF}$ are rising strikingly among patients $>65$ years. In the UK, in 2000, AF accounted for $1 \%$ of the total National Health Service expenditure, and AF-related stroke burden is calculated to cost US\$billions yearly, which is set to rise by its increased incidence in the elderly.

Second, there is need for improved understanding of the mechanisms of AF. About 30\% of AF cases occur in patients with no cardiopulmonary disease; in around 25\%, AF contributes to or results from heart failure, and the remainder arise in patients with hypertension, ischemic or valvular heart disease or diabetes. In patients with cardiopulmonary disease, myocardial remodeling secondary to the disease process or to the AF by itself results in the generation of areas of differential conduction and refractoriness or 'multiple wavelet', particularly when paroxysmal AF becomes persistent or permanent. In patients without cardiopulmonary disease, normal myocardium, local areas of inflammation and fatty or nonfatty infiltration

\section{The toll of atrial \\ fibrillation, particularly \\ on elderly \\ patients, and \\ its cost to \\ society are \\ incessantly \\ escalating}

$V$ Fuster is the Editorin-Chief of Nature

Clinical Practice

Cardiovascular

Medicine.

\section{Competing interests}

The author declared he has no competing interests.

www.nature.com/clinicalpractice doi:10.1038/ncpcardio0199 have been seen, suggestive of myocarditis or underlying metabolic, genetic or familial causes. In patients with paroxysmal AF and normal myocardium particularly, 'atrial focal firing' around the pulmonary vein orifices has also been observed, with or without vagal or sympathetic involvement. AF-associated thrombosis leading to stroke frequently arises in the left-atrial appendix, mainly due to stasis mechanisms, suggested by spontaneous echo contrast or 'smoke' as a result of fibrinogen-mediated red blood cell aggregation.

Third, management of AF is still challenging. Rate control might improve quality of life, but not survival or stroke prevention. If rate control fails, restoration of normal sinus rhythm is the longterm goal. If rhythm control is not deemed necessary initially in asymptomatic patients, achieving sinus rhythm can, however, become increasingly difficult with longer time elapsed, even with advancing catheter or surgical ablation technologies. Furthermore, in persistent or permanent $\mathrm{AF}$, symptoms can progressively arise related to structural or functional myocardial remodeling. Thus, assessment of the effect of AF on quality of life and optimum timing of therapy is needed.

Fourth, primary prevention of AF and related stroke can be rationalized by addressing those diseases associated with about two-thirds of cases. Prophylactic antiarrhythmic treatment before coronary artery bypass grafting can prevent postoperative AF, but is not usually indicated for a first episode of AF or infrequent, well-tolerated paroxysmal AF. A risk-stratification model has been proposed for the use of oral anticoagulants in primary prevention of AF-related stroke, but strategies are still needed to address related bleeding risks in elderly patients and the development of safer, more effective and easily administered fixed-dose antithrombotics.

In summary, the toll of AF, particularly on elderly patients, and its cost to society are incessantly escalating. Thus, this disorder still presents a formidable challenge to epidemiologists, scientists and clinicians. 